\title{
Susceptibilidad de híbridos de Populus spp. al ataque de áfidos y roya en tres localidades de Chile
}

\author{
Susceptibility of Populus spp. hybrids to aphids and rusts at three localities of Chile
}

\author{
María E Rubio-Meléndez a , Francisco Zamudiob ${ }^{\text {, }}$ Claudio C Ramírez ${ }^{\text {a,c* }}$ \\ ${ }^{a}$ Universidad de Talca, Instituto de Biología Vegetal y Biotecnología. \\ b Universidad de Talca, Facultad de Ciencias Forestales, Centro Tecnológico del Álamo, Talca, Chile. \\ *Autor de correspondencia: ' Universidad de Talca, Facultad de Ciencias Agrarias, Laboratorio de Sanidad Vegetal, 2 Norte 685, \\ Talca, Chile, tel.: 56-71-200289, clramirez@utalca.cl
}

\begin{abstract}
SUMMARY
In the last few years an increasing interest on species and hybrids of the genus Populus has occurred in Chile. However, such plantations are strongly affected by pest and diseases. With the aim to identify potentially resistant (or less susceptible) hybrids of the genus Populus, the susceptibility of some poplar hybrids growing in experimental nurseries in Southern-Center Chile to the aphid Chaitophorus leucomelas Koch and to the rust Melampsora spp. was assessed. Results showed that aphid abundance was higher in the month of March, particularly in Coinco (O’Higgins Region) and lower in Pillanlelbún (Los Ríos Regions) and Yumbel (Biobío Region). In Pillanlelbún the most susceptible hybrid to aphids was TD $\times$ TD $[(P$. trichocarpa $x$ P. deltoides $) \times(P$. trichocarpa $x$ $P$. deltoides $)]$, and the less susceptible ones were $\mathrm{TD} \times \mathrm{D}[(P$. trichocarpa $\times$ P. deltoides $) \times P$. deltoides $]$, $\mathrm{T} \times \mathrm{N}(P$. trichocarpa $x$ P. nigra), TMxTM [(P. trichocarpa x P. maximowitzii) x (P. trichocarpa x P. maximowitzii)] and TDxT [(P. trichocarpa $\times$ P. deltoides) $x$ $P$. trichocarpa]. Hybrid TxN (P. trichocarpa $x$ P. nigra) was the most susceptible to rust in all testing sites, while the less susceptible hybrids were TDxT $[(P$. trichocarpa $\times$ P. deltoides $) \times$ P. trichocarpa $]$, TDxD [(P. trichocarpa $x$ P. deltoides $) \times P$. trichocarpa $]$ and TDxT $[(P$. trichocarpa $\times$ P. deltoides $) \times P$. trichocarpa]. These susceptibilities varied among localities, particularly on aphids. Genetic and environmental factors accounting for by these results are discussed.
\end{abstract}

Key words: susceptibility, Chaitophorus leucomelas, Melampsora prevalence, abundance.

\section{RESUMEN}

En los últimos años se ha generado un creciente interés en el potencial forestal de las especies e híbridos del género Populus (álamos) en Chile. Sin embargo, algunas plagas y patógenos atacan severamente a estas plantaciones. Con el fin de identificar algunos híbridos resistentes (o menos susceptibles) a estas plagas, se evaluó la susceptibilidad de algunos taxones de Populus spp. al ataque del áfido Chaitophorus leucomelas y al hongo Melampsora spp. El estudio se realizó en tres localidades del centro-sur de Chile. Los resultados revelan que la densidad de áfidos fue mayor en marzo, particularmente en Coinco (región de O'Higgins) y menor en Pillanlelbún (región de Los Ríos) y Yumbel (región del Biobío). En Pillanlelbún el taxón más susceptibles a áfidos correspondió a TD $\times$ TD $[(P$. trichocarpa $\times$ P. deltoides $) \times(P$. trichocarpa $\times$. deltoides $)]$ y los menos susceptibles fueron $\mathrm{TD} \times \mathrm{D}[(P$. trichocarpa $x$ P. deltoides $) \times P$. deltoides $], \mathrm{T} \times \mathrm{N}(P$. trichocarpa $\times$ P. nigra $)$, TMxTM [(P. trichocarpa $\times$ P. maximowitzii $) \times(P$. trichocarpa $\mathrm{x}$ $P$. maximowitzii $)]$ y $\mathrm{TD} \times \mathrm{T}[(P$. trichocarpa $\times$ P. deltoides $) \times P$. trichocarpa $]$. Las infecciones con Melampsora spp. se presentaron en orden decreciente en Coinco, Yumbel y Pillanlelbún. El taxón $\mathrm{T} \times \mathrm{N}$ fue el más susceptible a roya en las tres regiones, mientras que los menos susceptibles fueron $\mathrm{TD} \times \mathrm{T}$, $\mathrm{TD} \times \mathrm{D}$ y $\mathrm{TD} \times \mathrm{T}$. Estas susceptibilidades variaron entre localidades, principalmente para áfidos. No hubo correlación entre la abundancia de C. leucomelas y de Melampsora spp. Se discuten los factores genéticos y ambientales que podrían explicar estos resultados.

Palabras clave: susceptibilidad, Chaitophorus leucomelas, prevalencia de Melampsora, abundancia.

\section{INTRODUCCIÓN}

En los últimos años se ha generado un creciente interés en el potencial forestal de las especies e híbridos del género Populus (álamos) para Chile. Su utilización podría contribuir a la solución de los problemas económicos del sector agrícola (baja rentabilidad de algunos cultivos) y forestal (monopolio de precios en productos). Dadas las capacidades fitoremediadoras de los híbridos del género
Populus, éstos podrían además ayudar a resolver problemas relacionados con la calidad del ambiente. En la actualidad, Chile cuenta con aproximadamente 6.500 hectáreas de plantaciones de híbridos de Populus spp., como por ejemplo Populus x canadensis Moench 'I-214' (P. deltoides Marshall. x P. nigra L.), Populus x canadensis Moench 'I-488' ( $P$. deltoides $x$ P. nigra), entre otros (Zamudio et al. 2002a). Los que se distribuyen, principalmente, entre las regiones del Maule y Libertador General Bernardo 
O’Higgins. Sin embargo, desde 1999 se han introducido a Chile más de 2.500 híbridos del género Populus. Esto ha significado un aumento en la diversidad genética del género en un $60 \%$ (Zamudio et al. 2002a). De esta forma, el país cuenta con una amplia base genética de híbridos de álamo, a partir de la cual es posible seleccionar aquellos que sean utilizables para plantaciones comerciales en aquellas áreas en donde Pinus radiata D. Don y las especies del género Eucalyptus no han podido establecerse o no han alcanzado su máximo desarrollo.

Los árboles de Populus spp. son atacados por una amplia diversidad de insectos y patógenos. Entre los híbridos actualmente cultivados en Chile se han detectado varias especies de insectos perforadores (Melanophila pictica Pallas (Coleptera: Buprestidae), Rhyephenes maiIlei (Gay et Solier) (Coleoptera: Curculionidae), Tremex fuscicornis Fabricius (Hymenoptera: Siricidae)), defoliadores (Macromphalia ancilla (Philippi) (Lepidoptera: Lasiocampidae), Ormiscodes cinnamomea (Feisthamel) (Lepidoptera: Saturnidae), Nematus desantisi Smith (Hymenoptera: Tenthredinidae)), chupadores (Phoeomyzus passerinii Sing, Pterocomma populeum (Kaltenbach) (Hemiptera: Aphididae), Diaspidiotus ancylus (Putman) (Hemiptera: Diaspididae), Chaitophorus leucomelas Koch (Hemiptera: Aphididae), Tuberolachnus salignus Gmel (Lachninae)) y agalladores (Pemphigus bursarius (Linneo)) (Sanhueza et al. 1998). También se han detectado algunos hongos, entre los cuales se destacan los pertenecientes a distintas especies del género Melampsora. Los álamos también son atacados por plantas parásitas, como el quintral (Phrygilantus tetrandus R. et Pay), que son muy abundantes en la zona de Colchagua, región Libertador General Bernardo O’Higgins. En las plantaciones comerciales y ornamentales de híbridos del género Populus es habitual apreciar el ataque del áfido Chaitophorus leucomelas (pulgón del álamo), así como de distintas especies del género Melampsora (roya). Estos agentes producen los principales daños en álamos en Chile (Ramírez et al. 2004).

Chaitophorus leucomelas se alimenta a través de la absorción de la savia de los álamos, parasitándolos y generándoles lesiones en el tejido foliar (Blackman y Eastop 1994, Sanhueza et al. 1998, Giganti et al. 2004). Dentro de los síntomas que presentan las plantas se destaca el amarillamiento de las hojas y su caída prematura (Giganti et al. 2004). La mielecilla secretada por estos áfidos provoca la aparición de hongos saprófitos (fumaginas) sobre las hojas, la que atrae a hormigas e insectos mutualistas (Salomon 1986, Giganti et al. 2004). Los daños que ocasionan son, generalmente, debilitamiento de las hojas, disminución de la capacidad fotosintética y reducción del crecimiento del árbol de hasta un 15 \% (Ramírez y Verdugo 2009). Los áfidos producen además, daños indirectos al constituir vectores de varios virus y bacterias (Sanhueza et al. 1998). Se ha descrito que los taxones de Populus ssp. introducidos a Chile que presentan mayor y menor susceptibilidad a C. leucomelas, son aquellos que incluyen en sus parentales a las especies Populus deltoides Marsh. y Populus maximowitzii Henry, respectivamente (Ramírez et al. 2004). Dado que hay evidencias en otras latitudes de que la susceptibilidad a plagas de Populus ssp. puede variar entre distintos sitios (Coyle et al. 2006, Tomescu y Nef 2007), es probable que tal situación también se presente en Chile.

Por otra parte, hongos del género Melampsora son causantes de las enfermedades de las hojas de los álamos, más importantes en el mundo. Alrededor de 13 especie de Melampsora spp. han sido descritas para Populus spp. (Pei y Shang 2005). Los huéspedes de la roya incluyen a coníferas, monocotiledóneas y dicotiledóneas. En estas últimas destacan especies de los géneros Salix (sauces) y Populus. En Chile, aparentemente la roya no desarrolla su ciclo sexual, posiblemente debido a que no existen cultivos del huésped secundario (Larix spp.), manteniéndose en receso en hojas y ramas caídas durante el periodo invernal. La presencia de roya en las hojas de los álamos depende de las condiciones climáticas (temperatura y humedad relativa) y disponibilidad de nitrógeno y potasio en el suelo durante el periodo de crecimiento de los híbridos, entre otros factores (Sanhueza et al. 1998). Por otra parte, el ataque de Melampsora spp. comienza a principios del verano, y su dispersión es facilitada por el viento o por material vegetal provenientes de otros sitios (Sanhueza et al. 1998, Cortizo 2003). Los primeros síntomas aparecen como pequeñas manchas verde amarillentas translucidas a contra luz, luego evolucionan transformándose en pústulas (uredosoros) amarillo anaranjado, las que pueden estar dispersas o agrupadas. En ataques intensos llegan a cubrir toda la superficie inferior de la hoja (Cortizo 2003). Los daños se basan, principalmente, en la defoliación de las plantas tanto en los viveros como en plantaciones, siendo en Europa Melampsora larici-populina Kleb. la responsable de la mayor parte de las pérdidas económicas en las plantaciones de álamo (Frey et al. 2005). A diferencia del caso de $C$. leucomelas, no hay estudios publicados en Chile respecto de la variación en susceptibilidad a Melampsora spp. de los taxones de Populus spp. Tampoco hay estudios de la variación geográfica de la susceptibilidad de Populus spp. a Melampsora spp. En otras latitudes se ha determinado que tal susceptibilidad es afectada por factores genéticos de Populus spp. (Newcombe et al. 1996), la severidad de los patotipos de Melampsora spp. (Pinon 1992, Frey et al. 2005) y las características de los plantaciones (Toome et al. 2010).

Las investigaciones sobre resistencia y susceptibilidad al ataque de patógenos pueden ser usadas como herramientas que orienten la toma de decisiones en cuanto al manejo de plantaciones forestales ya sean monoclonales o multiclonales. Pinon (1992) y Zamudio et al. (2002a) sugieren establecer rodales multiclonales de álamos y así crear cultivos forestales más estables, dejando de manifiesto que el establecimiento de grandes extensiones forestales 
monoclonales involucra un riesgo ecológico y económico.

El objetivo de este trabajo fue evaluar la susceptibilidad de siete taxones de especies del género Populus, a través de monitoreo de la abundancia del áfido C. leucomelas y del nivel de infección del hongo Melampsora spp., en ensayos clonales instalados en tres diferentes localidades de Chile. La hipótesis de trabajo indica que los taxones del género Populus presentan diferencias en su susceptibilidad a C. leucomelas y Melampsora spp., la que a su vez varía entre distintas localidades (sitios) de Chile central.

\section{MÉTODOS}

Sitios de estudio y material vegetal. Los taxones de Populus spp. monitoreados corresponden a plantas pertenecientes a tres ensayos experimentales del Centro Tecnológico del Álamo (CTA) de la Universidad de Talca instalados en tres localidades (cuadro 1), los cuales se encontraban espaciados a $1 \times 0,5 \mathrm{~m}$ en una superficie total de 0,5 hectáreas, en un arreglo tipo jardín común (Zamudio et al. 2002b), incluyendo doce cruzamientos interespecíficos. Estos taxones fueron introducidos a Chile entre agosto de 1999 y marzo de 2001 provenientes de Poplar Molecular Genetics Cooperative de la Universidad de Washington (Seattle, WA). El estudio se llevó a cabo entre diciembre 2005 y abril 2006 en tres viveros experimentales del Centro Tecnológico del Álamo en que se ensayó la adaptabilidad de varios clones y familias de varios taxones del género Populus, que incluyó un total de 4.600 árboles. Dada la imposibilidad logística de evaluar áfidos y royas en todos los árboles disponibles, se debió seleccionar un subconjunto de árboles para monitoreo que este trabajo se reporta. En cada localidad se seleccionaron árboles de la misma edad (2 años) pertenecientes a taxones que estuvieran presentes en las tres localidades y que además pertenecieran a familias y clones dentro de los taxones que también estuvieran presentes en las tres localidades. De esta forma, en cada localidad se seleccionó un total de 35 árboles, cinco por cada uno de los siete taxones de Populus spp., cada uno de familias distintas y sin replicas dentro de los clones por sitio de estudio (cuadro 2). Las variables respuestas consideradas en este estudio corresponden a densidad de áfidos y nivel de infección de roya.

Monitoreo de áfidos. En cada árbol se seleccionó una rama al azar ubicada en el primer tercio inferior del árbol. El monitoreo se realizó mediante muestreos mensuales, entre
Cuadro 2. Descripción de los taxones utilizados en el estudio. Description of taxa used in the study.

\begin{tabular}{ccc}
\hline $\begin{array}{c}\text { Progenitor } \\
\text { femenino }\end{array}$ & $\begin{array}{c}\text { Progenitor } \\
\text { masculino }\end{array}$ & Taxón \\
\hline $\mathrm{T}$ & $\mathrm{T}$ & $\mathrm{T} \times \mathrm{T}$ \\
$\mathrm{TM}$ & $\mathrm{TM}$ & $\mathrm{TM} \times \mathrm{TM}$ \\
$\mathrm{T}$ & n.i. & $\mathrm{T}$ \\
$\mathrm{TD}$ & $\mathrm{T}$ & $\mathrm{TD} \times \mathrm{T}$ \\
$\mathrm{TD}$ & $\mathrm{N}$ & $\mathrm{TD} \times \mathrm{N}$ \\
$\mathrm{TD}$ & $\mathrm{D}$ & $\mathrm{TD} \times \mathrm{D}$ \\
$\mathrm{TD}$ & $\mathrm{TD}$ & $\mathrm{TD} \times \mathrm{TD}$ \\
\hline
\end{tabular}

T: Populus trichocarpa. M: P. maximowitzii. D: P. deltoides. N: P. nigra. n.i.: no identificado (unidentified).

diciembre de 2005 y abril de 2006. Las mediciones fueron registradas desde la base de la rama seleccionada hasta su ápice, separando la rama en tres secciones: basal, media y distal. En cada sección se contabilizó el número de individuos presentes en ambas caras de cada hoja. Durante el conteo de individuos se identificaron morfos ápteros (ninfas y adultos) y alados. Una hoja representativa de cada sección fue fotografiada a fin de estandarizar la estimación de número de áfidos por área de hoja. Las fotografías incluyeron una base de papel milimetrado y fueron tomadas con una cámara digital. Posteriormente, a través del programa Sigma Scan versión 9.0, se obtuvo una estimación del área foliar para cada sección. La densidad de áfidos (individuos $/ \mathrm{cm}^{2}$ ) fue definida como el cociente entre el número de áfidos por hoja y el área foliar correspondiente. Se obtuvieron tres estimaciones de densidad de áfidos para cada árbol. Dado que no se detectó otra especie de áfido, este estudio sólo se refiere a C. leucomelas.

Monitoreo de roya. En la misma rama seleccionada de los árboles identificados para el monitoreo de áfidos se realizó el monitoreo del nivel de infección de roya. La evaluación de la enfermedad se realizó para cada hoja de la rama según una clasificación de ocho categorías establecidas relativa al porcentaje de ureosoros presentes (cuadro 3). Las mediciones se realizaron en los meses de marzo y abril de 2006, período donde se desarrolla la mayor parte de la roya.

Análisis estadísticos. Los análisis para la densidad de áfidos y el nivel de infección de roya se realizaron a través del programa Statistica versión 7.0. Dado que los datos

Cuadro 1. Descripción y ubicación de las localidades de estudio.

Description and locations of study sites.

\begin{tabular}{|c|c|c|c|c|}
\hline Localidad & Latitud (S); longitud (O) & $\begin{array}{l}\text { Altitud } \\
\text { (m s.n.m) }\end{array}$ & $\begin{array}{l}\text { Precipitación media } \\
\text { anual (mm) }\end{array}$ & $\begin{array}{c}\text { Temperatura media } \\
\text { anual }\left({ }^{\circ} \mathrm{C}\right)\end{array}$ \\
\hline Coinco & $34^{\circ} 14^{\prime} ; 70^{\circ} 04^{\prime}$ & 343 & 495 & 13,4 \\
\hline Yumbel & $37^{\circ} 13^{\prime} ; 72^{\circ} 34^{\prime}$ & 77 & 1.051 & 13,1 \\
\hline Pillanlelbún & $38^{\circ} 39^{\prime} ; 72^{\circ} 27^{\prime}$ & 159 & 1.389 & 11,6 \\
\hline
\end{tabular}


Cuadro 3. Clasificación de los niveles de infección con Melampsora spp. en hojas de Populus spp.

Classification levels of infestation by rust (Melampsora spp.) on the leafs (Populus spp.).

\begin{tabular}{cl} 
Nivel & Porcentaje de presencia de Melampsora spp. \\
\hline 0 & No hay infección \\
1 & Algunos soros vistos con dificultad \\
2 & Soros visibles pero muy esparcidos \\
3 & $25 \%$ de la superficie foliar atacada \\
4 & 25 a $50 \%$ de la superficie foliar atacada \\
5 & 50 a $75 \%$ de la superficie foliar atacada \\
6 & 75 a $100 \%$ de la superficie foliar atacada \\
7 & $100 \%$ hoja atacada con necrosis foliar \\
\hline
\end{tabular}

obtenidos no cumplieron los supuestos de normalidad, se aplicó la prueba de Scheirer-Ray-Hare (extensión de Kruskal-Wallis, Sokal y Rohlf 1995) para dos factores con medidas repetidas, con el fin de comparar entre taxones y localidades (factores: localidad y taxón) en los meses de muestreo (medida repetida: nivel de ataque o nivel de infección de roya). Las comparaciones dentro de las localidades entre meses de muestreo se realizaron mediante la prueba de Friedman, en tanto que las comparaciones entre taxones dentro de localidades se realizaron mediante Kruskal-Wallis, seguido de comparaciones múltiples. Finalmente, se analizó, mediante correlación simple la relación entre C. leucomelas y Melampsora spp. Para esto último, se correlacionó el promedio de áfidos versus el promedio del nivel de infección de roya para cada cruzamiento en las localidades estudiadas. Para este último caso, sólo se trabajó con los datos del mes de marzo debido a las bajas abundancias de Melampsora spp. en abril.

\section{RESULTADOS}

Densidad de áfidos. El análisis global de la densidad de áfidos mostró variación significativa entre los sitios en estudio (cuadro 4), con una mayor densidad en la localidad de Coinco y mayor abundancia en abril en Pillanlelbún (prueba de Kruskal-Wallis, $\mathrm{H}=57,4$; g.l. $=2$; $P<0,001$; figura 1). No se observó efecto global del taxón ni interacción localidad $x$ taxón. Se observó una variación significativa entre los meses (cuadro 4), con el mes de marzo con una mayor cantidad de ataque de áfidos (prueba de Friedman, $\chi^{2}=25,7$; g.l. $=3 ; P<0,00001$ ).

La densidad de áfidos también mostró una interacción significativa entre localidades y los meses de evaluación (cuadro 4), con una densidad máxima en febrero y marzo para Coinco (prueba de Friedman, $\chi^{2}=57,4$; g.l. $=4 ; P<$ $0,001)$ y abril para Pillanlelbún (prueba de Friedman, $\chi^{2}=$ 32,4; g.l. $=4 ; P<0,001$ ) (figura 1). Aun cuando el análisis global no detectó una interacción meses $\mathrm{x}$ localidades $\mathrm{x}$ taxón significativa, un análisis dentro de cada localidad mostró que sólo en el ensayo de Pillanlelbún, hubo una interacción meses $x$ taxón (prueba de Scheirer-Ray-Hare con medidas repetidas: $\mathrm{H}_{12,70}=22,9, P=0,028$ ) (figura 2), observándose que el taxón más susceptibles fue TD×TD [(P. trichocarpa x P. deltoides) $\times$ (P. trichocarpa $\times$ P. deltoides)], y una mayor presencia de C. leucomelas en el mes de abril.

Nivel de infección de roya. El análisis global reveló una significativa variación entre localidades, taxones, meses de muestreo y sus interacciones (cuadro 5). Se observó un mayor nivel de infección en Yumbel, en tanto que en las tres localidades estudiadas, marzo fue el mes con mayor infección. El taxón $\mathrm{T} \times \mathrm{N}$, (P. trichocarpa x P. nigra), fue el más susceptible a roya en las tres regiones, mientras que el taxón $(\mathrm{TD} \times \mathrm{T})[(P$. trichocarpa $\times$ P. deltoides $)$

Cuadro 4. Tabla ANDEVA de dos factores (localidad y taxón) con mediadas repetidas en cuatro meses (excluido diciembre) de la densidad de áfidos sobre siete cruzamientos de especies del género Populus.

Two-way ANOVA (location and hybrid) with repeated measures table in four months (excluded December) of aphid density on seven hybrids of the genus Populus.

\begin{tabular}{lrrrrr}
\hline \multicolumn{1}{c}{ Fuente de variación } & \multicolumn{1}{c}{ SC } & g.l. & CM total & SC/ CM total & \multicolumn{1}{c}{$P$} \\
\hline Localidad & 569.423 & 2 & & 47,34 & $<0,0001$ \\
Taxón & 95.351 & 6 & & 7,92 & 0,23 \\
Localidad x Taxón & 79.407 & 12 & & 6,60 & 0,88 \\
Error & 506.607 & 84 & & & \\
Total & 1.250 .788 & 104 & 12.026 & & $<0,0001$ \\
\hline Meses & 212.588 & 3 & & 24,35 & $<0,0001$ \\
Meses x Localidad & 618.816 & 6 & & 70,88 & 0,33 \\
Meses x Taxón & 181.165 & 18 & & 20,75 & 0,65 \\
Meses x Localidad x Taxón & 279.957 & 36 & & 32,07 & \\
Error & 1.457 .485 & 252 & & & \\
Total & 2.750 .011 & 315 & 8.730 & & \\
\hline
\end{tabular}




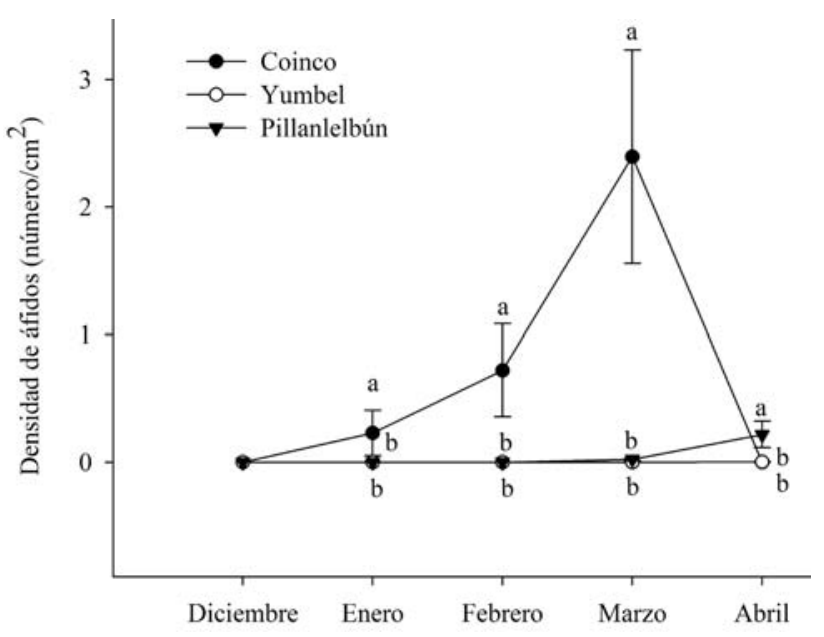

Figura 1. Densidad de C. leucomelas (media \pm error estándar) por mes de muestreo en Coinco, Yumbel y Pillanlelbún. Letras iguales dentro de los meses, indican ausencia de diferencias significativas luego de comparaciones múltiples de la prueba de Kruskal-Wallis.

Density of C. leucomelas (mean \pm standard error) for each sampled month in Coinco, Yumbel and Pillanlelbún. Similar letters within months, indicating no significant differences after multiple comparisons Kruskal-Wallis test.

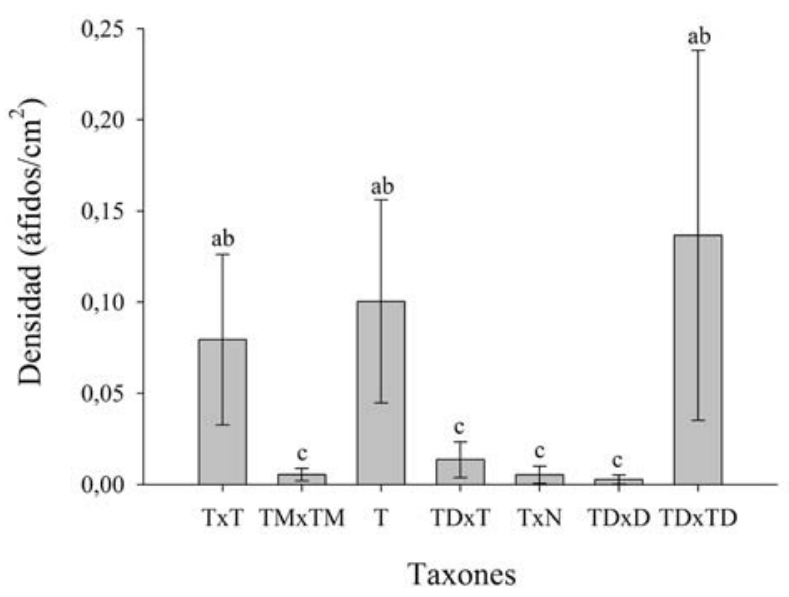

Figura 2. Densidad de C. leucomelas (media \pm error estándar) en cruzamientos de Populus spp. en Pillanlelbún en abril. Letras distintas sobre las barras indican diferencias significativas $(P \leq 0,05)$ entre medianas según prueba $\mathrm{Z}$ de comparaciones múltiples de Kruskal-Wallis.

Density of C. leucomelas (mean \pm standard error) by Populus spp. hybrids in Pillanlelbún in April. Different letters above bars indicate significant differences $(P \leq 0.05)$ among medians according to $\mathrm{Z}$ test for multiple comparisons Kruskal-Wallis.

$x$ P. trichocarpa], fue el menos susceptible. Al considerar la interacción localidades y los meses de registro, en Coinco no se observó un efecto significativo de los meses analizados (prueba de Scheirer-Ray-Hare con medidas repetidas; $\mathrm{H}_{1,70}=3,1 ; P=0,078$ ), aunque las mayores diferencias se obtuvieron en el mes de marzo (prueba de
Kruskal-Wallis: $\mathrm{H}_{6,266}=144,9 ; P<0,001$ ) (figura 3A). En dicha localidad hubo ausencia de diferencias entre los taxones para el mes de abril (prueba de Kruskal-Wallis: $\mathrm{H}_{3,57}=0,30 ; P=0,96$ ) (figura 3B). En el caso de Yumbel, se observó un efecto significativo de los meses analizados (prueba de Scheirer-Ray-Hare con medidas repetidas; $\left.\mathrm{H}_{1,532}=125,4 ; P<0,001\right)$. El nivel de infección de roya en Yumbel presentó diferencias significativas entre los taxones en el mes de marzo (prueba de Kruskal-Wallis: $\mathrm{H}_{6,266}=144,9 ; P<0,001$ ) (figura 3C), en tanto que en abril no se encontraron diferencias significativas entre los taxones (prueba de Kruskal-Wallis: $\mathrm{H}_{2,22}=2,1 ; P=0,35$ ) (figura 3D). Finalmente, en Pillanlelbún tampoco se observó un efecto significativo de los meses analizados (prueba de Scheirer-Ray-Hare con medidas repetidas: $\mathrm{H}_{1,472}=0,079$; $P=0,79)$. En esta localidad el nivel de infección de roya
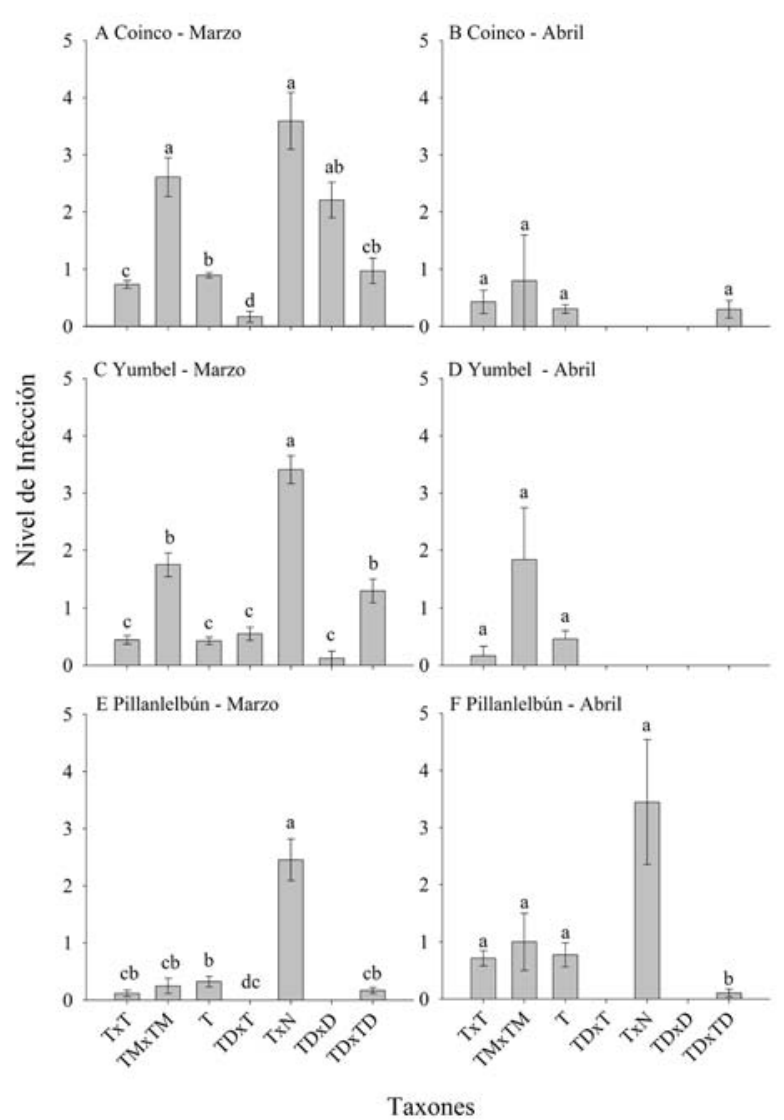

Figura 3. Comparación de los niveles de infestación de Melampsora spp. (media \pm error estándar) en los siete cruzamientos en los ensayos de Coinco, Yumbel y Pillanlelbún para los meses de marzo y abril. Letras distintas sobre las barras indican diferencias significativas $(P \leq 0,05)$ entre medianas según prueba $\mathrm{Z}$ de comparaciones múltiples de Kruskal-Wallis.

Comparison of infestation levels of Melampsora spp. (mean \pm standard error) on the seven Populus hybrids studied in the Coinco, Yumbel and Pillanlelbún in March and April. Different letters above bars indicate significant differences $(P \leq 0.05)$ among medians according to $\mathrm{Z}$ test for multiple comparisons Kruskal-Wallis. 
Cuadro 5. Tabla ANDEVA de dos factores (localidad y taxón) con mediadas repetidas (meses de marzo y abril) de la infección de roya en siete cruzamientos de especies del género Populus.

Two-way ANOVA (location and hybrid) with repeated measures table (March and April) of the rust infection on seven hybrids of the genus

Populus.

\begin{tabular}{lrrccc}
\hline \multicolumn{1}{c}{ Fuente de variación } & \multicolumn{1}{c}{ SC } & \multicolumn{1}{c}{ g.l. } & CM total & SC/ CM total & $P$ \\
\hline Localidad & 1.532 .186 & 2 & & 252,65 & $<0,001$ \\
Taxón & 2.301 .738 & 6 & & 379,54 & $<0,001$ \\
Localidad x Taxón & 1.839 .896 & 12 & & 303,39 & $<0,001$ \\
Error & 6.673 .399 & 2.016 & & & \\
Total & 12.347 .219 & 2.036 & $6.064,4$ & & $<0,001$ \\
\hline Meses & 4.159 .295 & 1 & & 511,66 & $<0,001$ \\
Meses x Localidad & 1.274 .500 & 2 & & 156,78 & $<0,001$ \\
Meses x Taxón & 2.550 .384 & 6 & & 313,74 & $<0,001$ \\
Meses x Localidad x Taxón & 1.628 .960 & 12 & & 200,39 & \\
Error & 6.945 .517 & 2.016 & & & \\
Total & 16.558 .656 & 2.037 & $8.128,9$ & & \\
\hline
\end{tabular}

presentó diferencias estadísticamente significativas tanto para el mes de marzo $\left(\mathrm{H}_{5,236}=111,37 ; P<0,001\right)$ (figura 3E) como para el mes de abril $\left(\mathrm{H}_{5,236}=52,16 ; P<0,001\right)$ (figura 3F).

Correlación entre abundancia de Chaitophorus leucomelas y Melampsora spp. En dos de las localidades estudiadas, la abundancia de C. leucomelas y Melampsora spp. mostró una correlación no significativa, pero con tendencia negativa (Coinco: $r=-0,41, P=0,35$; Pillanlelbún: $\mathrm{r}=-0,49, P=0,25)$. La localidad de Yumbel no fue correlacionada debido a la ausencia de áfidos.

\section{DISCUSIÓN}

Los resultados muestran que la densidad de C. leucomelas varió entre los diferentes sitios ensayados, lo que sustenta parcialmente la hipótesis de trabajo para el caso de esta plaga. La mayor abundancia de C. leucomelas en la localidad de Coinco podría explicarse por las altas tasas de crecimiento en los árboles ensayados, a pesar de constituir una zona de menores precipitaciones. Sin embargo, este ensayo se localizó muy cercano de la ribera del río Cachapoal pudiendo absorber agua desde su napa freática. Respecto de la variación en abundancia de C. leucomelas entre los taxones estudiados, los más susceptibles correspondieron al taxón TDxTD, registrados en Coinco. Sin embargo, los menos susceptibles pertenecieron a TDxD, TMxTM, TxN y TDxT. Un patrón de susceptibilidad a C. leucomelas similar fue explicado por Ramírez et al. (2004) para la localidad de Talca, quienes también describieron una mayor susceptibilidad para el taxón TDxTD y baja susceptibilidad para TMxTM.

Los resultados sugieren que la abundancia del áfido C. leucomelas sería más afectada por las características ecológicas de los sitios, más que por las características genéticas de resistencia de los árboles estudiados. Por ejem- plo, la mayor o menor abundancia de C. leucomelas en los sitios de estudio pudo verse afectada por la presencia de cultivos de álamos aledaños, los que habrían incidido en la probabilidad de arribo del áfido desde esas plantaciones a los sitos estudiados. Sin embargo, otros estudios plantean que la abundancia de áfidos puede ser afectada tanto por el genotipo de la planta (Hochwender y Fritz 2004, Johnson 2008) como por la interacción genotipo-ambiente (Paige y Capman 1993). Otro factor no excluyente con los anteriores, dice relación con la variación genética intraespecífica de las poblaciones de C. leucomelas en Chile, pudiendo existir biotipos con habilidades diferenciales para atacar los taxones de Populus spp. estudiados. Dado que durante el monitoreo de estos insectos se pudo constatar la presencia de individuos sexuales (machos alados y hembras sexuales ápteras), la componente de variación genética de la plaga pudo también ser importante. A este respecto, análisis genéticos de la composición clonal de las poblaciones de C. leucomelas podrían contrastar esta hipótesis.

Algo diferente se observó en la susceptibilidad a Melampsora spp. La susceptibilidad varió entre sitios y los taxones TDxT, TDxD y TDxT fueron los que se mostraron menos susceptibles en Coinco, Yumbel y Pillanlelbún, respectivamente. Sin embargo, el taxón TxN fue el más susceptible en los tres sitios de estudio. Los resultados de este trabajo indican que, a diferencia de la infestación con C. leucomelas, la susceptibilidad al ataque de Melampsora spp. parece no depender de las características ecológicas de los sitios estudiados, sino más bien al origen genético de los taxones analizados. Esta evidencia sustenta parcialmente la hipótesis de trabajo, pues por un lado se observó variación en la susceptibilidad a Melampsora spp. entre los taxones de Populus spp., pero por otro lado esa susceptibilidad no varió entre los sitios estudiados. Otros estudios en especies de salicáceas han mostrado que el nivel de infección de Melampsora spp. es principalmente dependiente del genotipo de la planta (Roche y Fritz 1998, Heiska et al. 
2007). Por ejemplo, en Chile la producción del género Salix en plantaciones comerciales está dominada por plantaciones monoclonales, lo que podría asociarse con un aumento en la variabilidad de la virulencia y la agresividad de los patotipos de Melampsora spp. presentes en Chile (Ramstedt y Hurtado 2005). Por otro lado, la baja variación geográfica de la abundancia Melampsora spp., particularmente la observada sobre el taxón TxN, podría explicarse por la predominancia de un patotipo particular. Son necesarios otros estudios que permitan dilucidar la variabilidad genética y patogénica de Melampsora spp. en Chile.

Los resultados sugieren que el ataque de áfidos estaría influenciado por las condiciones ambientales asociadas a las localidades de estudio. En localidades con mayor disponibilidad de recursos hídricos podrían desarrollarse árboles más vigorosos, los que se asociarían con mayor abundancia de insectos (Price 1991). En el caso de Melampsora spp., es probable que las condiciones climáticas no hayan sido un factor limitante para el desarrollo de la enfermedad. Sin embargo, se observó una tendencia a una mayor infección en aquellos taxones en cuyos parentales (padres) participan las especies Populus nigra y Populus maximowiczii. Se observa también que algunos pedigríes con taxón materno TD fueron menos susceptibles. Esta información podría ser relevante para futuros estudios en donde se evalúe tanto la genética de la planta como los patrones de infección de Melampsora spp. Dado que C. leucomelas y Melampsora spp. coexisten sobre las mismas plantas hospederas, se exploró la relación entre ambas especies, encontrándose una ausencia de correlación. Esta tendencia podría ser importante de evaluar en futuras investigaciones, con el fin de evaluar si existe una relación antagonista entre plaga-patógeno.

\section{CONCLUSIONES}

Los taxones del género Populus, que incluyen una parte importante de la variabilidad genética presente en Chile, muestran diferencias en su susceptibilidad al áfido C. leucomelas y Melampsora spp. La variación en la susceptibilidad a áfidos presenta una mayor variación entre sitios de estudio que entre taxones, en tanto que la susceptibilidad a Melampsora spp. presenta mayor variación entre taxones y una baja variación entre sitios de estudio. No hay evidencias de una asociación fuerte entre C. leucomelas y Melampsora spp.

\section{AGRADECIMIENTOS}

Los autores agradecen la colaboración en terreno de Hugo Pino, Jaime Verdugo, Marta Albornoz y Marcos Yáñez. Esta investigación contó con el financiamiento de FONDECYT 1040675. Además, del apoyo entregado por el Centro Tecnológico del Álamo de la Universidad de Talca y de los proyecto FONDEF D01I1131 y D04I1027.

\section{REFERENCIAS}

Blackman RL, VF Eastop. 1994. Aphids on the world's trees: An identification and information guide. Wallingford, U.K. Cab International. 987 p.

Cortizo S. 2003. Roya del álamo en el Delta del Paraná. Idia 5:135-138.

Coyle DR, MD Coleman, JA Durant, LA Newman. 2006. Multiple factors affect pest and pathogen damage on 31 Populus clones in South Carolina. Biomass \& Bioenergy 30(8-9): 759-768.

Frey P, P Gérard, N Feau, C Husson, J Pinon. 2005. Variability and population biology of Melampsora rusts on poplars. In Pei M, A McCracken eds. Rust diseases of willow and poplar. Wallingford, U.K. Cab International. p. 63-72.

Giganti HE, GLDapoto, MADelfino. 2004. Chaitophorus leucomelas Koch (Hemiptera, Aphididae) en Río Negro y Neuquén (Argentina). Características morfológicas y biológicas. Revista de Investigación Agropecuaria 33(2): 27-38.

Heiska S, OP Tikkanen, M Rousi, S Turtola, V Tirkkonen, B Meier, R Julkunen-Tiitto. 2007. The susceptibility of herbal willow to Melampsora rust and herbivores. European Journal of Forest Pathology 118(3): 275-285.

Hochwender CG, RS Fritz. 2004. Plant genetic differences influence herbivore community structure: evidence from a hybrid willow system. Oecologia 138(4): 547-557.

Johnson MTJ. 2008. Bottom-up effects of plant genotype on aphids, ants, and predators. Ecology 89(1): 145-154.

Newcombe G, HD Bradshaw, GA Chastagner, RF Stettler. 1996. A major gene for resistance to Melampsora medusae $f s p$ deltoidae in a hybrid poplar pedigree. Phytopathology 86(1): 87-94.

Paige KN, WC Capman. 1993. The effects of host-plant genotype, hybridization, and environment on gall-aphid attack and survival in cottonwood: The importance of genetic studies and the utility of RFLP. Evolution 47(1): 36-45.

Pei M, Shang Y. 2005. A brief summary of Melampsora species on Populus. In Pei M, A McCracken eds. Rust diseases of willow and poplar. Wallingford, U.K. Cab International. $280 \mathrm{p}$.

Pinon J. 1992. Variability in the genus Populus in sensitivity to Melampsora rusts. Silvae Genetica 41(1): 25-34.

Price P. 1991. The plant vigor hypothesis and herbivore attack. Oikos 62(2): 244-251.

Ramírez CC, JA Verdugo. 2009. Water availability affects tolerance and resistance to aphids but not the trade-off between the two. Ecological Research 24(4): 881-888.

Ramírez CC, F Zamudio, JA Verdugo, ME Núñez. 2004. Differential susceptibility of poplar hybrids to aphid Chaitophorus leucomelas (Homoptera: Aphididae). Journal of Economic Entomology 97(6): 1965-1971.

Ramstedt M, S Hurtado. 2005. Melampsora willow rust in Chile and northern Europe: Part of a metapopulation? In Pei M, A McCracken eds. Rust diseases of willow and poplar. Wallingford, U.K, Cab International. 280 p.

Roche BM, RS Fritz. 1998. Effects of host plant hybridization on resistance to willow leaf rust caused by Melampsora sp. European Journal of Forest Pathology 28(4): 259-270.

Salomon J. 1986. Early impact and control of aphid (Chaitophorus populicola Thomas) infectations on young cottonwood plantations en the Mississippi Delta. New Orleans, USA. USDA Forest Service. 4 p. 
Sanhueza A, M Bourke, F Schultz. 1998. Cultivo del álamo (Populus spp.). Santiago, Chile. CONAF. 131 p.

Sokal RR, FJ Rohlf. 1995. Biometry: the principles and practice of statistics in biological research. 3th ed. New York, USA. Freeman. 887 p.

Tomescu R, L Nef. 2007. Leaf eating insect damage on different poplar clones and sites. Annals of Forest Science 64(1): 99-108.

Toome M, K Heinsoo, B Holm, A Luik. 2010. The influence of canopy density on willow leaf rust (Melampsora epitea) severity in willow short rotation coppice. Biomass \& Bioenergy 34 (8): 1201-1206.

Zamudio F, I Chacón, F Guerra. 2002a. Estrategia de mejoramiento del género Populus en Chile. Monografía técnica. Talca, Chile. Universidad de Talca. 152 p.

Zamudio F, F Guerra, A Vergara, M Yáñez, J Pinon. 2002b. Crecimiento y adaptabilidad de nuevos híbridos de álamo a las condiciones locales de Talca, VII Región, Chile. Monografía técnica. Talca, Chile. Universidad de Talca. 54 p.

Recibido: 27.07.10

Aceptado: 22.03.11 